\title{
Leiomyoma of the soft palate: Case report and review of articles
}

\author{
Fahd Alharbi ${ }^{1,2}$
}

Case

Report

\author{
${ }^{1}$ Otorhinolaryngology-Head \& Neck Surgery Department, Faculty of Medicine, Jazan \\ University, Jazan, Saudi Arabia \\ ${ }^{2}$ Otorhinolaryngology-Head \& Neck Surgery Department, Prince Mohammed bin Nasser \\ Hospital, Jazan, Saudi Arabia
}

\begin{abstract}
Leiomyomas are benign tumors arising from smooth muscle, most commonly seen in uterine myometrium, gastrointestinal tract, skin and lower extremities of middle-aged women. Leiomyomas are uncommon in the oral cavity and leiomyomas of head and neck region account for less than $1 \%$ of all leiomyomas. The purpose of this article is to present a case of 18 years old male patient with a history of several months of oral leiomyoma on the right side of the soft palate. The clinical features, differential diagnosis and management of leiomyoma are discussed with review of the literatures.
\end{abstract}

Key Words: Benign, hard palate, leiomyoma, smooth muscle

Received: 15 October 2017, Accepted: 9 January 2018

Corresponding Author: Fahd Ali Alharbi, M.D., Faculty of Medicine, Jazan University, Saudi Arabia, Tel.: 00966543043452, E-mail: fahdalharbi3@gmail.com

ISSN: 2090-0740, March 2018 Vol.19, No.1

\section{INTRODUCTION}

Leiomyoma is a benign tumor arising from smooth muscle. Occurrence of leiomyoma in the head and neck regions is rare as these areas lack smooth muscle. Leiomyoma should be differentiated from connective tissue tumors such as lipoma and fibromas which may have similar findings. Also, it must be differentiated from leiomyosarcoma. Malignant salivary gland tumors must be included in the differential diagnosis ${ }^{[1]}$. The most common sites of leiomyoma of the head and neck were the lips, tongue, and hard and soft palate. More than 40\% of leiomyomas presented as an intraoral lesion, and more than half of the cases were presented for more than one year ${ }^{[2]}$. Histopathological findings play a key role in the final diagnosis of leiomyoma. At present, surgical resection is the treatment of choice for leiomyoma and recurrences are extremely rare.

\section{CASE REPORT:}

18 years old male patient presented with gradual swelling in the right side soft palate for several months was examined. On examination, a solitary swelling was noted on the right side of the posterior palate measuring
$2.5 \mathrm{~cm}$ by $1.5 \mathrm{~cm}$. Overlaying mucosa was pale pink in color aappear normal. The swelling was firm, dome shaped mass fixed to the underlying tissues. (Figure 1)

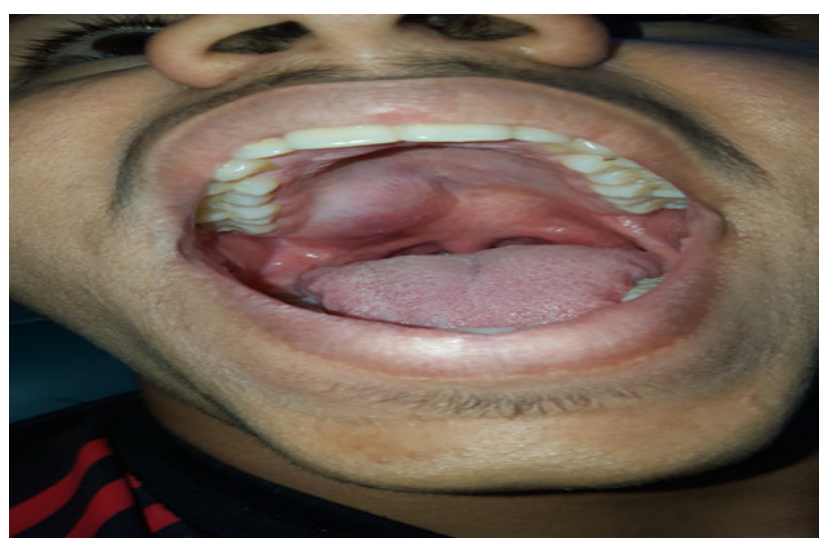

Fig. 1: A photograph of the oral cavity, showing a swelling in the area of the hard palate with extension to the soft palate right side

Magnetic resonance imaging (MRI) of the paranasal sinus showed intermediat signal solid mass lesion within right side of the soft palate and adjecent part of right hard palate on T1 weighted image. A T2 weighted image shows fairly incresed signal intensity of the previously described mass lesion (Figures 2 A, 2 B) 


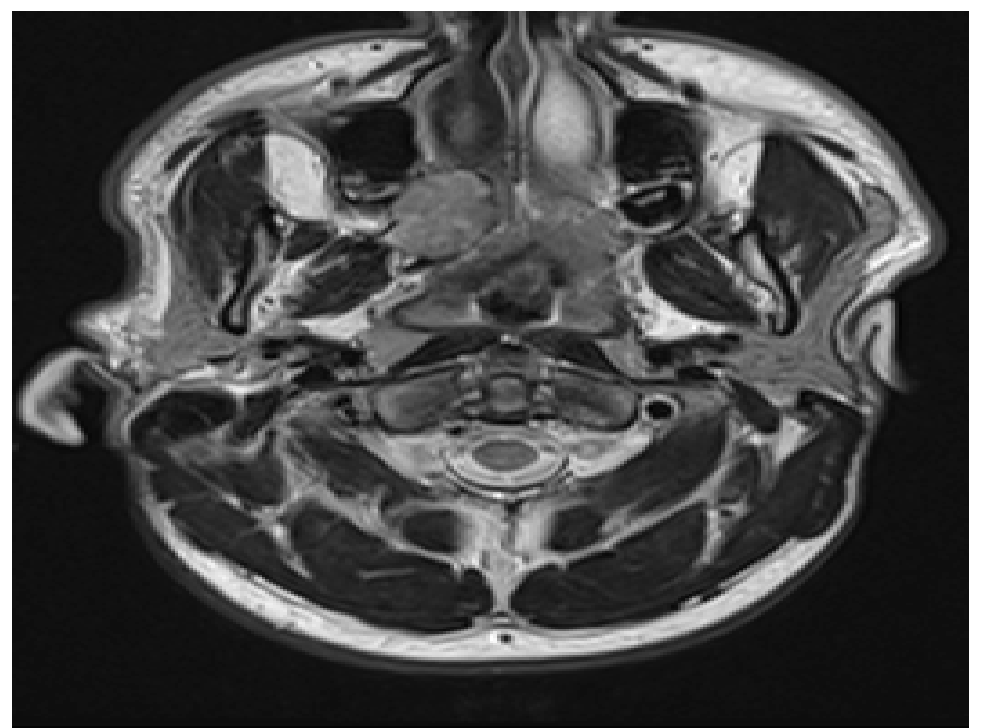

Fig. 2 A: Axial T2-weighted image showing a uniform signal and hypertense lesion on the right side of the hard palate.

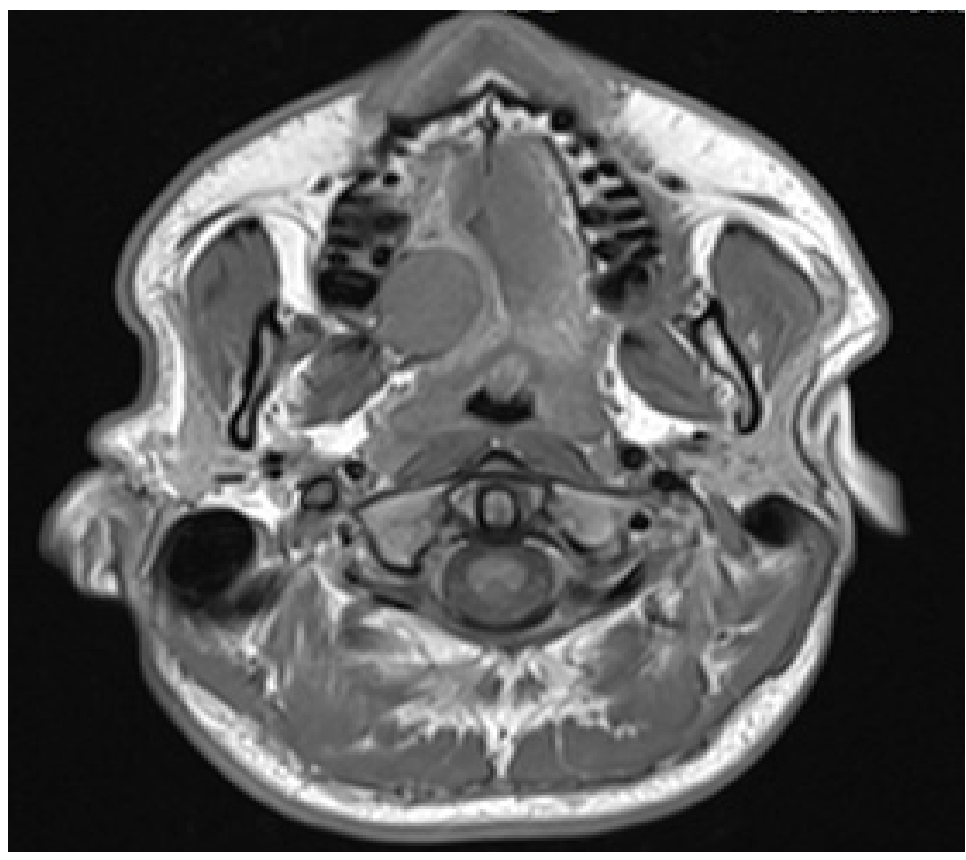

Fig. 2 B: Axial T1-weighted image showing the enhancement pattern and relation to the intraoral tissue of the hard palate.

T1-weighted image with intravenous gadolinium contrast media shows nearly homogenous enhancement pattern with no definit invasive pattern to adjecent structures, suggestive of benign nature mass lesion (Figures 3 A, 3 B, 3 C).

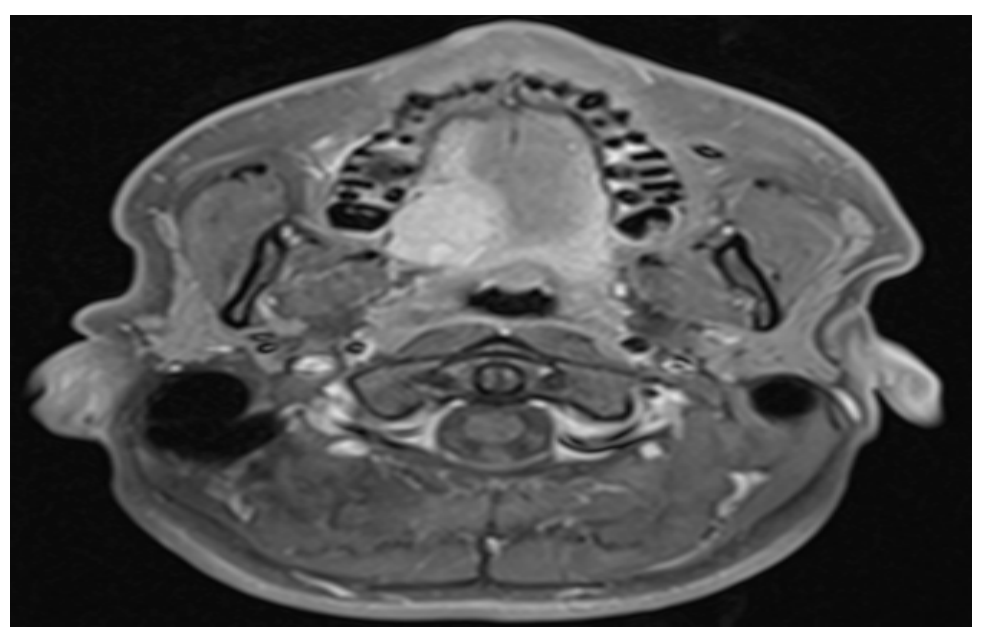

Fig. 3 A: Axial T1-weighted image study with I.V gadolinium contrast media shows nearly homogenous enhancement pattern with no definit invasive pattern to adjecent structures,suggestive of benign nature mass lesion.

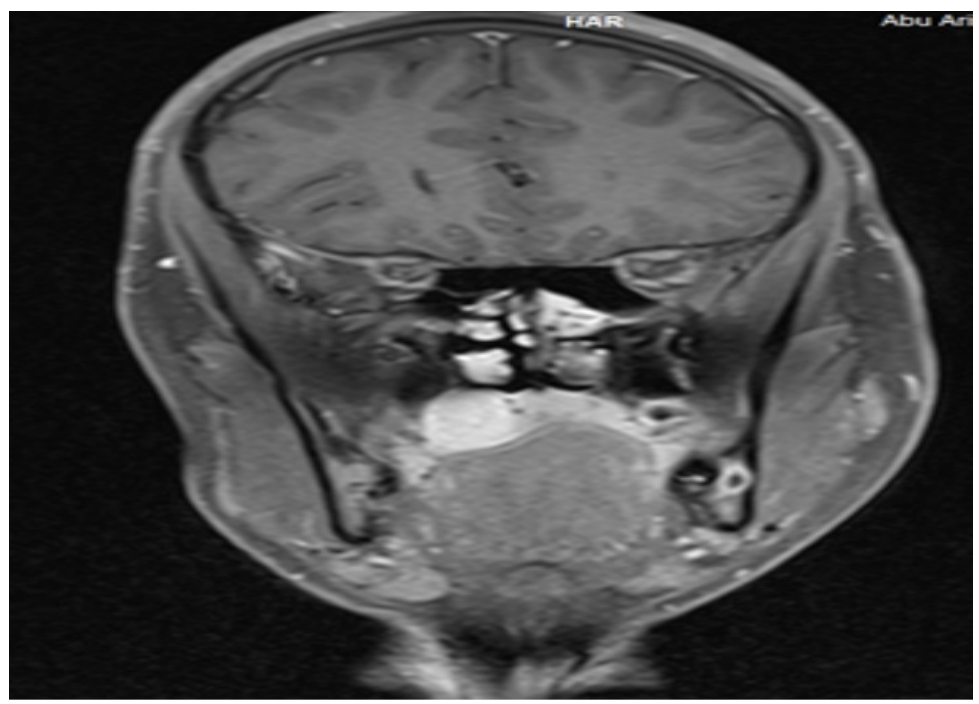

Fig. 3 B: Coronal T1-weighted image study with I.V gadolinium contrast media shows nearly homogenous enhancement pattern with no definit invasive pattern to adjecent structures,suggestive of benign nature mass lesion.

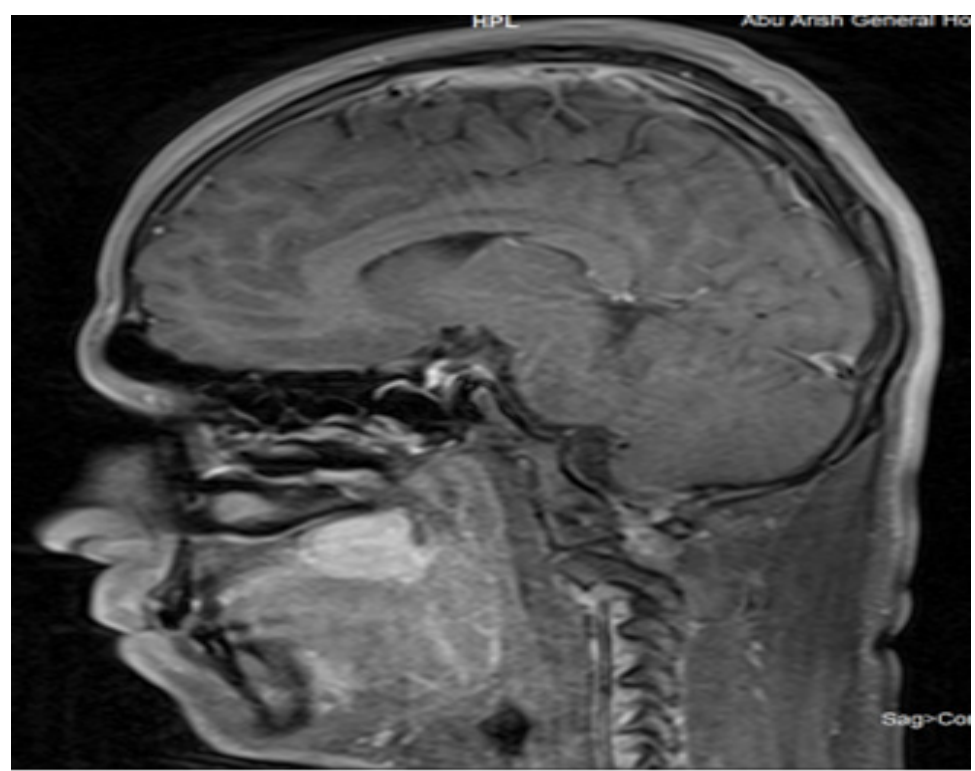

Fig. 3 C: Sagittal T1-weighted image study with I.V of gadolinium contrast media shows nearly homogenous enhancement pattern with no definit invasive pattern to adjecent structures,suggestive of benign nature mass lesion.

Fine needle aspiration was performed which ruled out a vascular lesion and repeated several times and was inconclusive regarding cytological examination. The lesion was provisionally diagnosed as a minor salivary gland tumor. The patient underwent complete transoral tumor excision of the mass with no intraoperative complication (Figure 4).

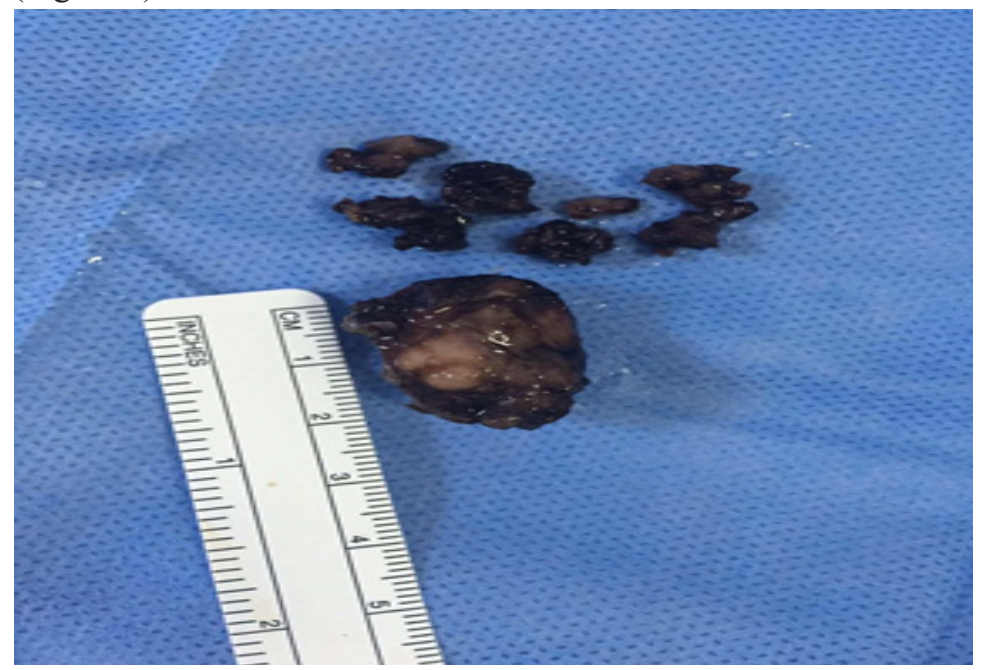

Fig. 4: A photograph showing the resected surgical specimen. 
The wound was closed mainly primarily after undermining the mucosa and small area left to heal by secondary tension. The postoperative period was without complications with regular wound healing.

Histopathology examination hematoxylin and eosin stained slides showed neoplasm formed of interlacing fascicles of spindle cells. The cells were arranged in whorls and fascicles (Figure 5).

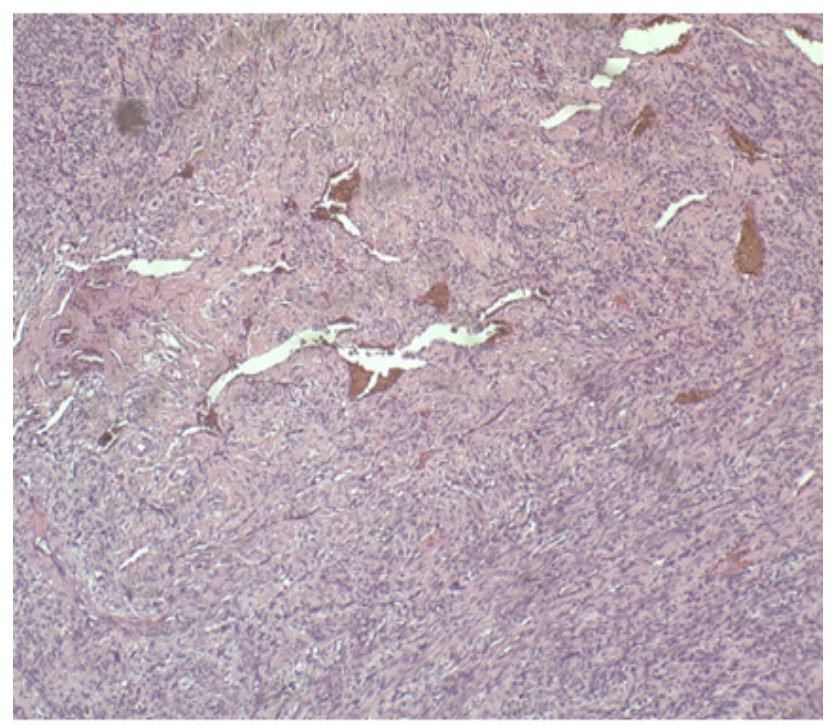

Fig. 5: The micrograph shows the whorly " fascicular pattern" of the neoplasm, made of smooth muscle bundles with numerous vascular spaces. " $\mathrm{H} \& \mathrm{E} 10 \mathrm{x}$ "

The smooth muscle cells appear elongated with eosinophilic and occasional fibrillar cytoplasm and distinct cell membranes. (Figure 6).

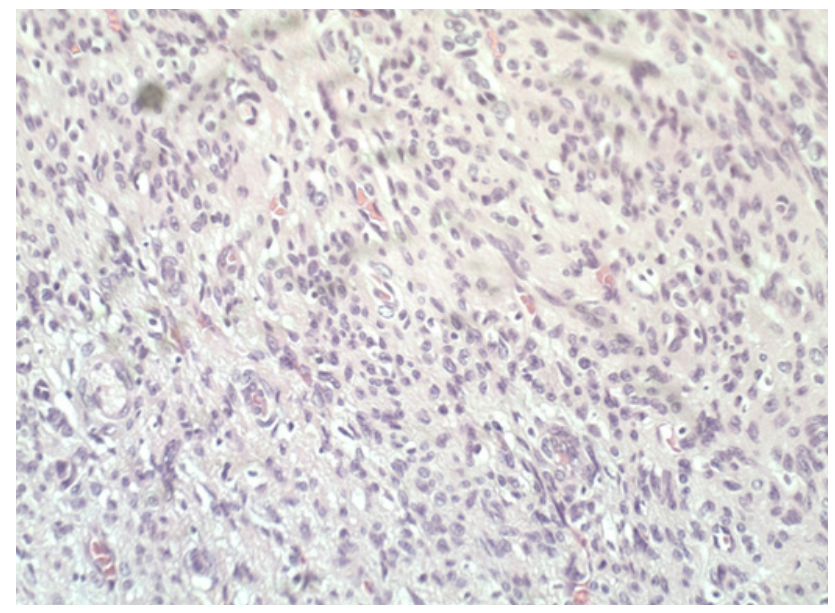

Fig. 6: The micrograph shows smooth muscle cells with elongated nuclei with distinct fibrillar cytoplasm " $\mathrm{H} \& \mathrm{E}$ x 40"

There are areas of degeneration, including hyaline or mucoid changes, calcification, cystic change or fatty metamorphosis variable lymphocytes and mast cells are also seen (Figure 7).

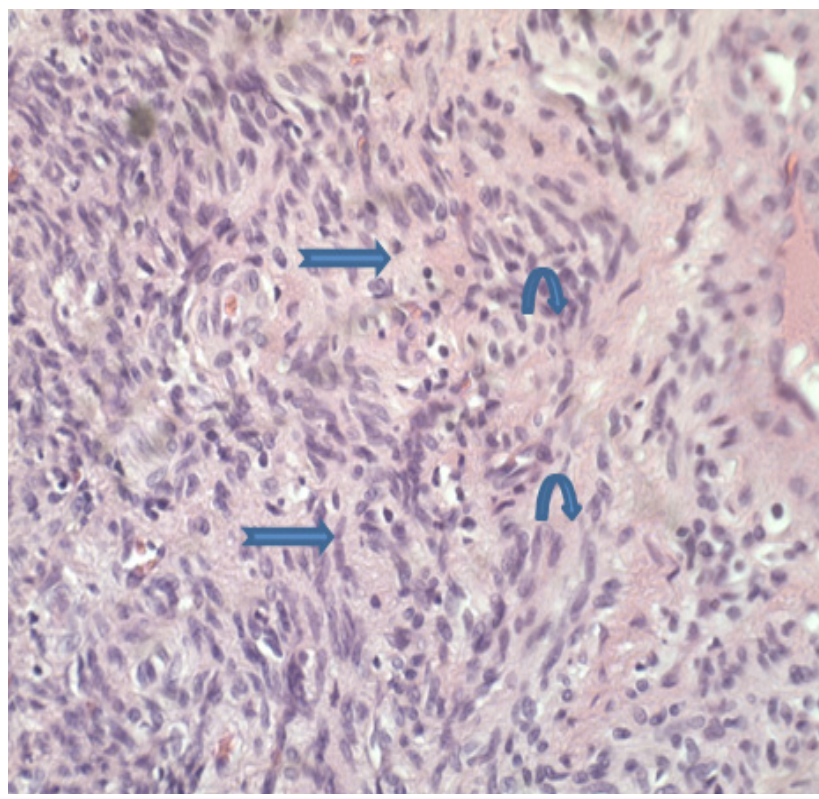

Fig. 7: The micrograph shows worly tumor pattern with some hyalinization of the stroma " curved arrows" with blood vessles and some lymphocytes. " arrows". "H\&E 10x"

Mitotic figures are seen (two mitosis/10 HPF). Minor salivary gland tissue is seen with bony tuberculae. The follow-up of the patient for one year showed no feature $\mathrm{s}$ of recurrence with complete wound healing.

\section{DISCUSSION}

Leiomyomas are benign tumors of smooth muscle. Oral leiomyoma is more common in male than female with peak age incidence of fourth decade of life for benign form and fifth decade for malignant tumors ${ }^{[3]}$. Most cases of leiomyomas in the oral cavity showed asymptomatic slow growing firm lesion, although occasionally, these tumors can be painful. In our case, the tumor was solitary 2.5 by $1.5 \mathrm{~cm}$ and has history of several months. The definitive diagnosis of leiomyoma must be made by histopathological diagnosis. Immuohistochemical studies differentiated from other spindle cell tumors can be helpful. Oral leiomyomas are classified as solid leiomyomas, vascular leiomyomas and epitheloid leiomyomas ${ }^{[4]}$. Malignant lesions such as leiomyosarcoma must be excluded as both can demonstrate a similar clinical features. The presence of atypia, cellularity, pleomorphism, necrosis and mitotic activity indicate malignant potential ${ }^{[5]}$. Kumar and Cotran mentioned that histological features of malignant changes include more than 10 mitosis/10 high-power fields (HPF) with or without cellular atypia and 5-10 mitosis/10 HPF with atypia. Tumors having 1-4 mitosis are considered as potentially malignant, especially if they are large and include areas of necrosis and have significant nuclear atypia ${ }^{[6]}$. In our case, mitotic activities were 2 mitosis/10 HPF and no malignant changes are detected. The treatment 
of choice is wide surgical resection with adequate resection margins. Recurrence following complete resection is rare. The prognosis of oral leiomyoma is excellent. The followup of our patient showed no signs of recurrence.

\section{CONCLUSION}

Leiomyoma is benign tumor with rare incidence in the area of head and neck region. The definitive diagnosis is mainly made by histopatholoigcal study. The treatment of choice is surgical resection. Leiomyoma has a good prognosis.

\section{CONFLICT OF INTEREST:}

There are no conflict of interest

\section{REFERENCES}

1. Veeresh M, Sudhakara M, Girish G, Naik C. Leiomyoma: A rare tumor in the head and neck and oral cavity: Report of 3 cases with review. J Oral Maxillofac Pathol. 2013 May;17 (2):281-7.
2. Wertheimer-Hatch L, Hatch GF $3^{\text {rd }}$, HatchB S KF, Davis GB, Blanchard DK, Foster RS Jr, Skandalakis JE. Tumors of the oral cavity and pharynx. World J Surg. 2000 Apr;24(4):395-400.

3. Gurkiran, Ranjana Gondal, Oral Leiomyoma, J OralMaxillofac Pathol. 2011 Sep-Dec; 15(3): 361-362.

4. Baden E, Doyle JL, Lederman DA, Leiomyoma of the oral cavity: a light microscopic and immunohistochemical study with review of the literature from 1884 to 1992 . Eur J Cancer B Oral Oncol. 1994;30B:1-7

5. Brooks JK, Nikitakis NG, Goodman NJ, Levy BA. Clinicopathologic characterization of oral angioleiomyomas. Oral Surg Oral Med Oral Pathol Oral Radiol Endod. 2002;94:221-7

6. Kumar V, Cotran RI. Robbin's Basic Pathology. $7^{\text {th }}$ ed. Philadelphia: Elsevier; 2004. P. 1090 